\title{
Quasi-Steady Analysis of a PBL Model with an Eddy-Diffusivity Profile and Nonlocal Fluxes
}

\author{
BJORN STEVENS* \\ Advanced Study Program, National Center for Atmospheric Research, ${ }^{+}$Boulder, Colorado
}

(Manuscript received 18 August 1998, in final form 22 February 1999)

\begin{abstract}
Analytic solutions to a planetary boundary layer (PBL) model with an eddy-diffusivity profile (i.e., a $K$ profile) and nonlocal fluxes are presented for the quasi-steady regime. The solutions demonstrate how different processes contribute to the quasi-steady profiles of heat and/or other scalars in the convective boundary layer. It is shown that for a standard cubic form of the $K$ profile, and flux scales based on the surface fluxes, the nondimensional nonlocal term should be less than six; larger values can cause scalar profiles of water vapor to increase with height in the upper portion of the PBL and can produce weakly superadiabatic layers in the upper PBL temperature profiles. Solutions are also shown to be sensitive to the choice of flux scale: fluxes scaled by their vertically averaged values imply that nondimensional profiles of top-down scalars will have a neutral point somewhere in the PBL, a result in conflict with previous work on the subject, and the predictions of the same model with fluxes scaled by their surface values. The analysis also shows that allowing $K$ to go to zero with the square of the distance from the PBL top results in nonconvergent profiles; in general $K$ should reduce to some positive value at the top of the PBL, or go to zero less rapidly. It is further shown that the class of models investigated here may be physically interpreted as relaxation models, that is, they tend to relax profiles of scalars in the PBL to implicitly defined similarity profiles on a convective timescale. Finally, analysis of a 1-yr integration of a climate model, interpreted in light of the author's analytic results, suggests that a dynamically important aspect of the nonlocal term is its role in ventilating the surface layer, and thereby indirectly affecting the diagnoses of PBL depth in many models.
\end{abstract}

\section{Introduction}

Ertel (1942) proposed that the potential temperature flux, $\overline{w \theta}$, be constituted of two terms, one proportional to the average lapse rate at the same height, the other not:

$$
\overline{w \theta}=-K_{h} \frac{\partial \Theta}{\partial z}+N
$$

The first term on the right is the traditional mixing length term, in that the gradient of the expected value of the potential temperature $\Theta(z, t)$ in a layer is multiplied by

\footnotetext{
* Current affiliation: Department of Atmospheric Science, University of California, Los Angeles, Los Angeles, California.

+ The National Center for Atmospheric Research is sponsored by the National Science Foundation.

Corresponding author address: Bjorn Stevens, Department of Atmospheric Science, University of California, Los Angeles, 405 Hilgard Ave., Los Angeles, CA 90095.

E-mail: bstevens@atmos.ucla.edu
}

a turbulent diffusivity, $K_{h}$, to give a flux. ${ }^{1}$ Ertel introduced the second term to account for the fact that in a convective layer, parcels crossing a level $z$, but originating at some level $z+l$, would tend to have a slightly elevated (for $l<0$ ) or depressed (for $l>0$ ) potential temperature relative to $\Theta(z+l, t)$; the main point being that this perturbation is not related to the local gradient. The addition of the convective term, to account for biased starting plumes, could then generalize the classical mixing-length theory to allow the local flux to be counter to the local gradient. ${ }^{2}$

Figure 1 presents a mean profile of potential temperature calculated on the basis of a large-eddy simulation (LES) of the convective atmospheric boundary layer. This profile illustrates the motive force behind (1), in that the flux and the local gradient are not generally proportional. Although our point is demonstrated on the basis of LES, this is done for illustrative purposes only-that there are regions in the PBL where the flux

\footnotetext{
${ }^{1}$ Throughout we distinguish between the expected value of a dependent variable at some level $z$, and the fluctuating component of that variable, through the use of upper and lower case, respectively.

${ }^{2}$ This theory is often attributed to Priestley and Swinbank (1947), who independently arrived at similar conclusions.
} 


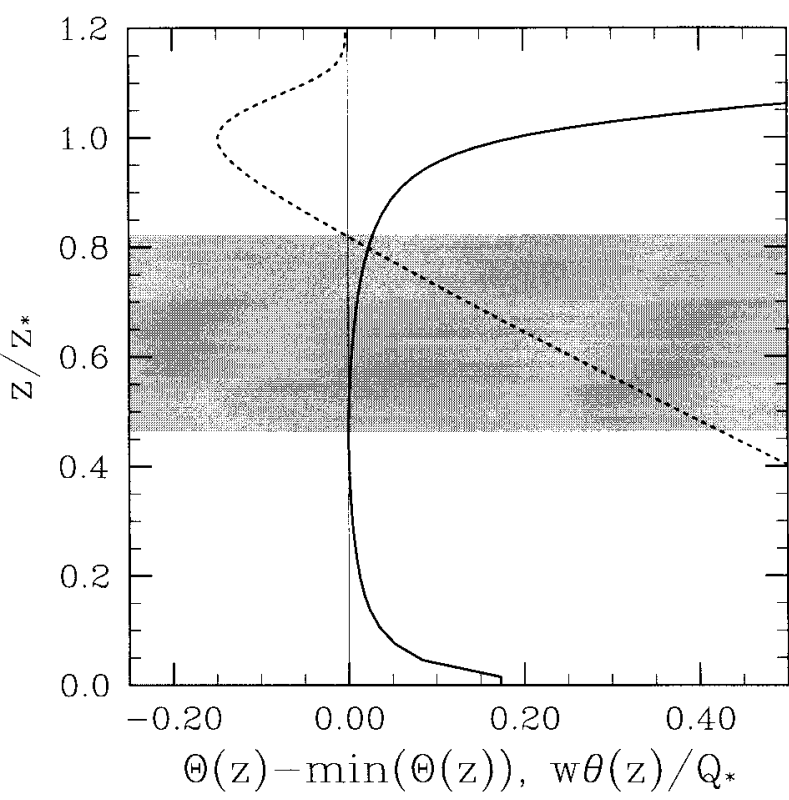

FIG. 1. Potential temperature $\Theta(z)$ minus its minimum value (thick solid line), and normalized flux, $\overline{w \theta} / Q_{*}$ (short dashed line), taken from a large-eddy simulation of the convective boundary layer. Shaded zone highlights the region where the flux of $\theta$ is counter to the gradient of $\Theta$. The neutral point $z_{n}$ of the profile is located at the base of the countergradient zone.

is counter to the local gradient has been accepted to be characteristic of real convective flows for at least a half a century (cf. Ertel 1942; Priestley and Swinbank 1947; Sutton 1953; Priestley 1959; Deardorff 1966).

Deardorff (1966), apparently unaware of Ertel's paper, proposed a model very similar to (1):

$$
\overline{w \theta}=-K_{h}\left(\frac{\partial \Theta}{\partial z}-\gamma_{h}\right),
$$

and used prior observations to estimate the value of the nonlocal term denoted by $\gamma_{h}$ above. Models of the flux having the form of (2) have since been incorporated into a variety of PBL models beginning with the model of Mailhôt and Benoit (1982). Subsequently, Troen and Mahrt (1986) used this nonlocal formulation of the fluxes in a model that diagnoses the PBL depth and then constrains $K$ (the eddy diffusivities or viscosities) to a fixed profile over the depth of the PBL. Variants of the $K$-profile approach of Troen and Mahrt have since been used in a wide variety of applications, such as climate studies (Holtslag and Boville 1993), weather forecast models (Beljaars and Betts 1992; Hong and Pan 1996), mesoscale models, and models of the upper-ocean boundary layer (Large et al. 1994). Consequently (2), with $K$ specified according to a fixed profile, shall form the basis for our subsequent study.

In the more than 30 years since (2) was proposed by Deardorff, a number of attempts have been made to formally justify $\gamma_{h} \neq 0$ on the basis of an analysis of budgets of the second-order equations (e.g., Deardorff

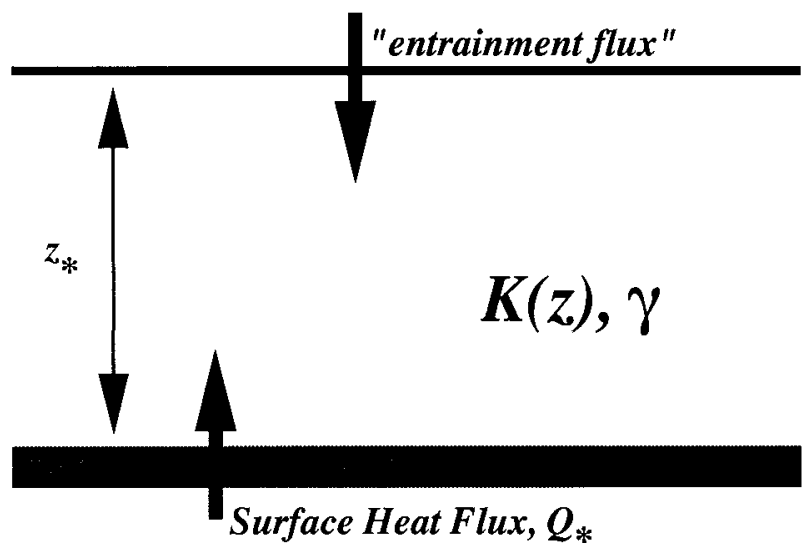

FIG. 2. Schematic illustrating the configuration of our problem, in which we heat a horizontally homogeneous box of fluid at the top and bottom.

1966, 1972; Holtslag and Moeng 1991). However, we know of no analysis that thoroughly shows how different values of $\gamma_{h}$ affect the quasi-steady solutions derived from PBL models that use (2) as a model of the flux. The point of this paper is to address this situation within the context of a specific type of PBL model, namely, the $K$-profile model. We take a predominantly mathematical point of view, and in so doing make no attempt to either physically justify the validity of (2) or assess its merits relative to competing theories of convective boundary layers - although physical issues raised on the basis of our analysis are highlighted in the last section of the discussion. For the most part, we simply wish to understand, in some general yet quantitative sense, the implications of allowing $\gamma_{h}$ to differ from zero, in part because we believe that a thorough mathematical understanding of (2) is a prerequisite to well-grounded physical interpretations of its effects. A further benefit of our approach is that it provides a simple framework within which we can rigorously address numerical convergence of our solutions as a function of the model discretization.

\section{Problem formulation}

A simple problem that allows us to gain insight into the effect of the nonlocal term is that of the evolution of the height-dependent expected value of the potential temperature in a convective layer bounded by two plates (separated by a distance $z_{*}$ ), and forced by a surface heat flux $Q_{0}$ and a constant "entrainment flux" at the upper boundary (e.g., Fig. 2). ${ }^{3}$ Strictly speaking entrainment also causes a deepening of the layer. To simplify

\footnotetext{
${ }^{3}$ We note that in so formulating the problem, we completely avoid one of the more important and interesting problems in the study of planetary boundary layers, namely how to determine the height and entrainment flux at the top of the PBL.
} 
the mathematics, this aspect of the problem has been neglected here, although numerical tests show that for a known entrainment flux this simplification has no notable effect on our analysis. Indeed, neglecting the deepening of the layer due to entrainment corresponds to the limiting solution of the problem as the capping inversion becomes infinitely strong, or the solution corresponding to the situation where large-scale subsidence exactly balances entrainment.

For a dry Boussinesq fluid in the absence of any mean wind, diabatic processes, or horizontal heterogeneity, the equation that governs the behavior of $\Theta(z, t)$ is simply,

$$
\frac{\partial \Theta}{\partial t}=-\frac{\partial \overline{w \theta}}{\partial z},
$$

which, upon application of our model for the flux, that is, (2), takes the form

$$
\frac{\partial \Theta}{\partial t}=\frac{\partial}{\partial z}\left[K_{h}\left(\frac{\partial \Theta}{\partial z}-\gamma_{h}\right)\right] .
$$

Equating a convective-flux scale, $Q_{*}$, with the surface heat flux (i.e., $Q_{*}=Q_{0}$ ), we can define a convective velocity scale $w_{*}=\left(g \beta Q_{*} z_{*}\right)^{1 / 3}$ [where $\beta=(1 / 300)$ $\mathrm{K}^{-1}$ is the coefficient of thermal expansion]. This definition of $w_{*}$ leads to the definition of an overturning (or convective) timescale, $t_{*}=z_{*} / w_{*}$, and a temperature scale $\theta_{*}=Q_{*} / w_{*}$, which together facilitate the nondimensionalization of our problem through

$$
\begin{array}{rlrl}
z \rightarrow z_{*} \hat{z}, \quad t \rightarrow t_{*} \hat{t}, & \Theta & \rightarrow \theta_{*} \hat{\Theta}, \quad \text { and } \\
K_{h} \rightarrow\left(w_{*} z_{*}\right) \hat{K}_{h}, & \gamma_{h} \rightarrow\left(\frac{Q_{*}}{w_{*} z_{*}}\right) \hat{\gamma}_{h} .
\end{array}
$$

Equation (4) may thus be interpreted either dimensionally or nondimensionally. Unless otherwise stated we adopt the nondimensional viewpoint, although in the interests of notational simplicity, we subsequently drop the hats that we have just now introduced. When we return to a dimensional framework in section 2 d, we distinguish dimensional variables by a superscript $d$, that is, $K_{h}^{d}$ refers to the dimensional diffusivity, similarly $z^{d}$ and $\gamma_{h}^{d}$ are used to represent the dimensional counterparts of $z$ and $\gamma_{h}$.

We are interested in quasi-steady solutions to (4). By quasi-steady solutions we mean steady-state solutions to

$$
\frac{\partial}{\partial t}\left(\frac{\partial \Theta}{\partial z}\right)=\frac{\partial^{2}}{\partial z^{2}}\left[K_{h}\left(\frac{\partial \Theta}{\partial z}-\gamma_{h}\right)\right],
$$

that is solutions of (4) that are independent of height. Consequently these solutions can be thought of as a class of similarity solutions to (4) in that they describe solutions for which $\partial \Theta / \partial z$ is invariant; that is, the shape of the $\Theta$ profile is not changing in time.

The assumption of quasi-steadiness is greatly simplifying, but not overly limiting, in that most of the

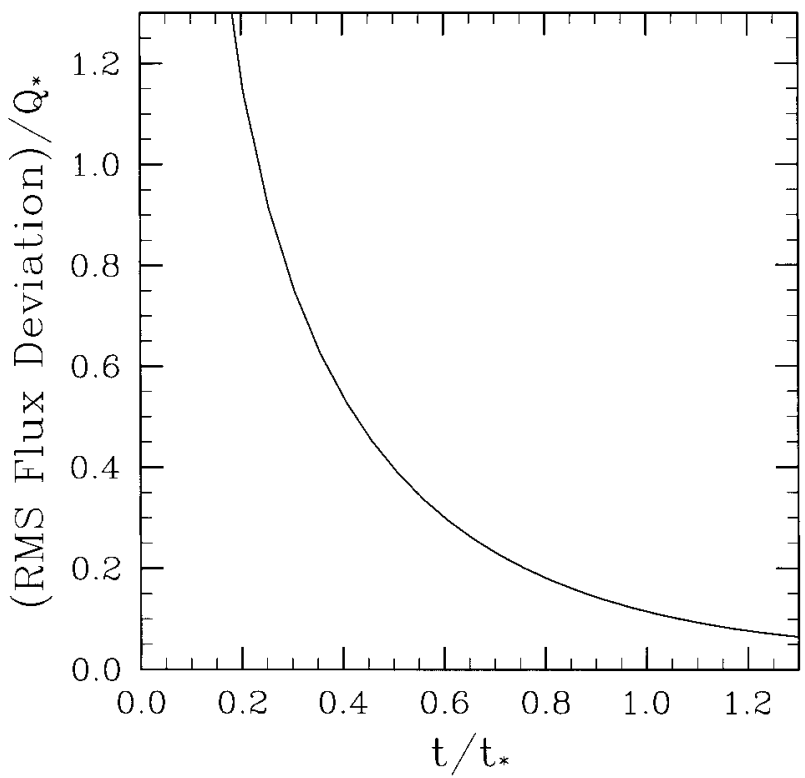

FIG. 3. Rms deviation of the flux at $t / t_{*}$ from its quasi-steady value for a numerical integration of (4) [with $K$ and $\gamma$ specified as in Holtslag and Moeng (1991)] in which initially the upper part of a $2.5-\mathrm{km}$ convective layer was warmed by $2 \mathrm{~K}$ and the lower part cooled by $2 \mathrm{~K}$ to ensure large deviations at the initial time.

analysis upon which (2) is based has been developed by studying LES in a quasi-steady state (cf. Holtslag and Moeng 1991; Cuijpers and Holtslag 1998). Further justification for this assumption is provided in Fig. 3, which shows that the rms deviation between the instantaneous and quasi-steady flux approaches zero on a timescale given by $t_{*}$. For a convective layer $t_{*}$ is about $10 \mathrm{~min}$. That means that the quasi-steady solutions are directly relevant to models with time steps greater than 10 min (e.g., most NWP and climate models), and indirectly relevant to models that consider higher-frequency phenomena, for example, regional and cloudresolving models, because [as our analysis in section $4 d(1)$ shows] on shorter timescales, the model effectively relaxes the profiles to their quasi-steady shapes on a convective timescale.

\section{Analytical solutions}

Steady-state solutions to (7) [or alternatively quasisteady solutions to (4)] are characterized by linear fluxes, that is,

$$
\bar{w} \theta / Q_{*}=-K_{h}\left(\frac{d \Theta}{d z}-\gamma_{h}\right)=(1-z)+A z,
$$

where $A$ is defined to be the ratio of the entrainment to the surface (or scaling) flux, and is taken as a parameter of our system. The dependency of the system on the surface flux is eliminated through the nondimensionalization (i.e., by writing $Q_{*}$ as a function of $Q_{0}$ ). Also, in seeking quasi-steady solutions, we have effectively 
removed the time dependence from the problem, so partial derivatives go over to total derivatives. Solving (8) for $d \Theta / d z$ results in

$$
\frac{d \Theta}{d z}=\frac{\gamma_{h} K_{h}(z)-[(1-z)+A z]}{K_{h}(z)} .
$$

If $\gamma_{h}$ and $K_{h}(z)$ take suitably simple forms, (9) can be integrated analytically. Although, for many purposes, a great deal of insight as to the nature of the quasisteady profiles of $\Theta$ can also be obtained from a simple consideration of the neutral points $z_{n}$ (e.g., Fig. 1). These points are here defined to be the level (or levels) where $d \Theta / d z$ vanishes; that is,

$$
\left.\frac{d \Theta}{d z}\right|_{z=z_{n}}=0 .
$$

Independent of the form of $\gamma_{h}$ and $K_{h}$, (9) illustrates how $z_{n}$ (or the $z_{n} \mathrm{~s}$ ) is determined by the surface fluxes, the entrainment fluxes (the $A$ term), and the product $\gamma_{h} K_{h}$. Because $\gamma_{h}$ and $K_{h}$ appear only together in the form of a product in the numerator of (9), and thus should not be specified independently, was noted by Troen and Mahrt (1986). It means that it is not possible to regulate the nonlocal fluxes by specifying $\gamma_{h}$ independently of $K_{h}$. So, for instance, values of $\gamma_{h}$ found to work well in one model, cannot necessarily be expected to work in a model that uses a different form of $K_{h}$. In a quasi-steady state one way to specify $\gamma_{h}$ would be as a function of $A$, the desired level of the neutral point, $z_{n}$, and $K_{h}$ :

$$
\gamma_{h}=\frac{\left(1-z_{n}\right)+A z_{n}}{K_{h}\left(z_{n}\right)} .
$$

a. Analytic $\Theta(z)$ profiles for common forms of $K_{h}$ and

$\gamma_{h}$

For $z>0.1, \gamma_{h}$ [denoted by $c_{4}$ in Holtslag and Moeng (1991), by $C^{\prime}$ in Troen and Mahrt (1986), and by $C_{*}$ in Large et al. (1994)] is typically given a constant value between 2 and 10 (Holtslag and Moeng 1991), and $K_{h}$ is given a prescribed shape

$$
K_{h}(z)=\kappa z(1-z)^{2} \quad z \in(0,1)
$$

chosen to reflect the shape of the vertical velocity variance profile (see also Cuijpers and Holtslag 1998). Specifying the maximum value $K_{h}$ is allowed to take, that is,

$$
K_{h}^{\uparrow}=\max _{0 \leq z \leq 1} K_{h}(z),
$$

determines the proportionality constant $\kappa$ (not to be confused with $k$, which we later introduce to represent von Kármán's constant). Analysis of LES by Holtslag and Moeng (1991) suggests that $K_{h}^{\uparrow}=0.1$, which implies $\kappa$ $=0.675$.

Before proceeding, some comments are in order regarding the form of (12). Equation (12) implies that $K$ vanishes at the boundaries of the turbulent flow. This implies unphysical gradients in the resultant profiles [cf. Eq. (9)] near $z=0$ and $z=1$. In principle $K$ should be reduced to a value no smaller than the maximum of either the molecular diffusivities (or viscosities) appropriate to the fluid under consideration, the values thought to best characterize the weak turbulent diffusivities (or viscosities) of the outer fluid in which the PBL is embedded (e.g., Large et al. 1994), or the value necessary to give the proper entrainment flux at $z_{*}$ (e.g., Beljaars and Betts 1992). Last, we note that in our problem, the entrainment zone has been approximated as infinitely thin. In reality (and especially for weakly capped flows) the heat flux only gradually approaches zero above the level of its minimum (cf. Fig. 1 where the flux only becomes zero at about $1.15 z_{*}$ ), neglecting the effects of a finite entrainment-zone depth in our problem is relevant to models that do not resolve such features; nonetheless, our prime motivation for this assumption is that it greatly simplifies the analysis but does not compromise the integrity of the analytic solutions away from the boundaries.

Substituting (12) into (9) yields

$$
\begin{aligned}
\frac{d \Theta}{d z} & =\frac{\gamma_{h} \kappa z(1-z)^{2}-[(1-z)+A z]}{\kappa z(1-z)^{2}} \\
& =\gamma_{h}-\frac{1}{\kappa z(1-z)}-\frac{A}{\kappa(1-z)^{2}},
\end{aligned}
$$

which lends itself to straightforward integration:

$$
\begin{gathered}
\Theta(z)=\gamma_{h} z-\frac{1}{\kappa} \ln \left(\frac{z}{1-z}\right)-\frac{A}{\kappa(1-z)}+\text { const. } \\
z \in(0,1) .
\end{gathered}
$$

Equation (16) shows how under (2), with $K$ given by (12) and $\gamma_{h}$ equal to a constant, the quasi-steady heat flux profile can be thought of as being determined by three processes (see also Fig. 4): surface fluxes (as illustrated by the term marked with a $B$ in the figure), entrainment (the $A$ term), and nonlocal processes proportional to $\gamma_{h}$. It follows from (14) that surface fluxes alone (e.g., without nonlocal fluxes) imply that $\Theta(z)$ is strictly decreasing with height throughout the PBL. Entrainment fluxes (with $A<0$ ) allow the gradient of the profile to vanish, but require that it does so only where the flux vanishes [as is obvious from (2)], while the nonlocal term allows the zero flux point and the neutral point to be at different levels. The constant of integration is determined by conservation of energy and thus reflects the time integrated heat input into the layer.

Unfortunately, the behavior of $\Theta\left(z ; \gamma_{h}, \kappa, A\right)$ is not obvious simply by inspecting (16); however, a rather simple way to gain insight into $\Theta\left(z ; \gamma_{h}, \kappa, A\right)$ is to consider the neutral points, $z_{n}$, of the $\Theta$ profile (recall that $z_{n}$ is any point where $d \Theta / d z$ vanishes). Although the neutral points do not uniquely determine the profile, they do place strong constraints on it. The locations of 


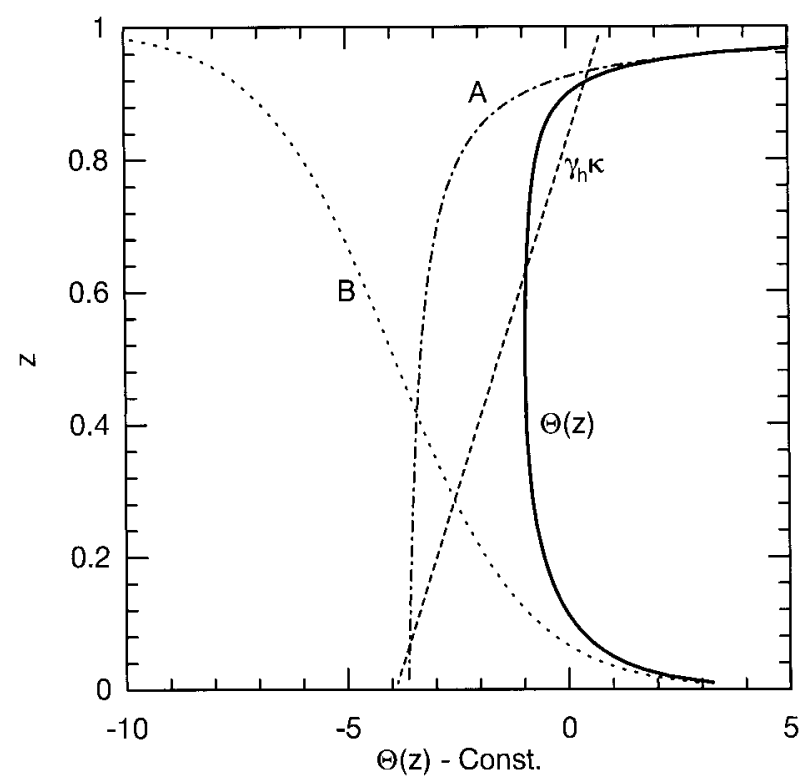

FIG. 4. Breakdown of different components of the analytic solution given by the thick solid line. Dotted line, surface processes. Short dashed line shows nonlocal term. Dashed-dotted line shows entrainment term. The $K$ terms tend to dilate along the $\theta / \theta_{*}$ axis for decreasing $\kappa$ while the nonlocal term's slope is given by $\kappa \gamma_{h}$. When interpreting near-boundary gradients in $\Theta / \theta_{*}$, recall that $\theta_{*}$ is typically about 0.2 , which tends to exaggerate the gradients in the profiles relative to scalings based on the strength of the inversion.

the $z_{n}$ are determined by demanding that the numerator of (14) vanish, that is,

$$
\begin{aligned}
f_{1}(z) & =\gamma_{h} \kappa z^{3}-2 \gamma_{h} \kappa z^{2}+\left(\gamma_{h} \kappa-A+1\right) z-1 \\
& =0 .
\end{aligned}
$$

Further insight into the mathematical properties of the solution is readily obtained through a thorough analysis of $f_{1}\left(z ; A, \gamma_{h} \kappa\right)$; although, we will not belabor this approach here, we do consider the solutions $f_{1}(z)$ $=0$ as a function of $A$ and $\gamma_{h} \kappa$. Figure 5a shows how $z_{n}$ varies with $\gamma_{h} \kappa$ for a range of values of $A$. For now we are interested in the $\Theta$ profile, so we only consider the curves corresponding to $A<0$. In this, $A<0$, regime there must always be a zero of $f_{1}$ (i.e., a place where the $d \Theta / d z$ vanishes) for $z \in(0,1)$; physically this makes sense because in the convective boundary layer the surface layer must be superadiabatic and the entrainment layer must be subadiabatic, hence, by continuity, at some point the profile must be neutral. The left-most point of the curves (i.e., $\gamma_{h} \kappa=0$ ) simply illustrates where the linear-flux is zero for a given value of $A$.

Two interesting features of the $A<0$ curves in Fig. 5a merit further discussion: (i) the implication that there is only a rather limited range of values for $\gamma_{h} \kappa$ capable of producing physical profiles; and (ii) that even within this range, that is, for reasonable values of $\gamma_{h} \kappa$, unphysical profiles can still result if the entrainment flux ratio $A$ becomes too small.
With respect to our point (i) above, Fig. 5b shows three curves for $A=-0.2$ but different values of $\gamma_{h} \kappa$. This figure, and panel (a) both suggest that value of $\gamma_{h} \kappa$ between 3 and 3.5 produce profiles, which by the location of their neutral points, seem to most closely correspond to LES (e.g., Fig. 1). These values corresponds to $4.4<\gamma_{h}<5.2$ for $K$ given by (12) and $\kappa=0.675$. For $\gamma_{h} \kappa<2.5$ the nonlocal model is having too small of an effect, and the region of countergradient flux is probably too small. For $\gamma_{h} \kappa>4.5$ the nonlocal flux is probably too dominant, leading to fluxes that already begin to become countergradient below $0.3 z_{*}$.

With respect to our point (ii) above, we note that if $A$ is too small, that is, in situations of very small entrainment fluxes the profile can have three zeros. In this situation there must be a region near the upper boundary of the flow where the gradient due to the surface flux dominates (because $A \ll 1$ ), forcing the profile to actually become weakly superadiabatic in the region between the second and third zero. This unsatisfactory aspect of the model-derived profiles is illustrated in Fig. 5c. Although we illustrate our point for the somewhat large value of $\gamma_{h} \kappa=4.8$, similar, albeit less pronounced, behavior can occur for more physical values of $\gamma_{h} \kappa$.

Last, we illustrate in Fig. 5d, a point noted above (that the values of $z_{n}$ do not uniquely determine the structure of the profile). Here we see that keeping $\gamma_{h} \kappa$ fixed while varying $\kappa$ can lead to profiles that are better mixed through a deeper layer (for larger $\kappa$ ), thus implying less superadiabatic profiles near the top of the surface layer.

\section{b. Solutions for passive scalars}

In addition to the heat flux, nonlocal fluxes are often included in evolution equations for other scalars such as moisture, or chemical constituents, in which case $A$ may take on a much wider range of values. If these scalars (generically referred to by $C$ hereafter) are conserved, then they too satisfy (4), except that $\Theta$ is replaced by $C, \overline{w \theta}$ is replaced by $\overline{w c}$, and the nondimensionalizing parameter $C_{*}$ is given by $\overline{w c}(z=$ $0) / w_{*}$ (where $w_{*}$ is still scaled based on the surface heat flux).

Thus all of our previous analysis for the quasi-steady $\Theta$ profiles extends directly to a consideration of the quasi-steady $C$ profiles, except that now we must consider a broader range of values for $A$. In particular, it is not unusual for the entrainment moisture flux to exceed the surface moisture flux. Figure 5a illustrates that as $A$ increases, increasingly large values of $\gamma_{c}$ are necessary to obtain a vanishing gradient in the solution for $z \in$ $(0,1)$. Although the initial tendency is for increasing $\gamma_{c}$ to lead to better mixed quasi-steady profiles in the PBL interior-as, for instance, is illustrated by the quasisteady profiles of $C$ plotted in Fig. 6 for $A=0.5$ and three different values of $\gamma_{c} \kappa$, this can be over done- 

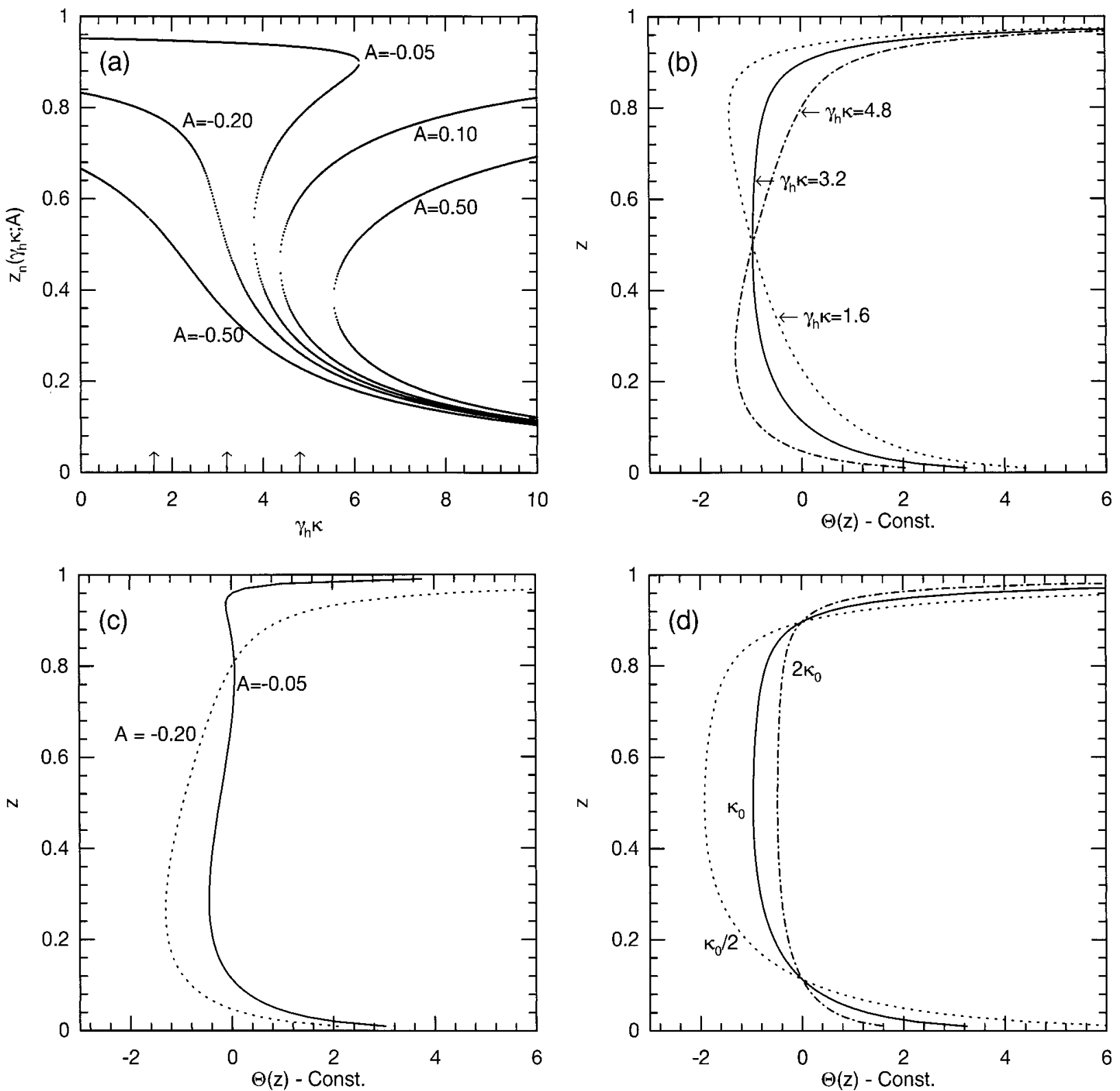

Fig. 5. (a) Solutions to (17) for different values of $A$ as labeled. (b) Analytic (nondimensional) profiles for $A=-0.2, \kappa=0.675$, and $\gamma_{h} \kappa$ varying as indicated. Note that these profiles correspond to the points marked by the arrows along the $\gamma_{h} \kappa$ axis in panel (a). (c) Profiles at $\gamma_{h} \kappa=4.8$ for two different values of $A$. (d) Profiles for $\gamma_{h} \kappa=3.2$ but $\kappa$ either doubled or halved, where $\kappa_{0}=0.675$.

too large a value of $\gamma_{c} \kappa$ can lead to a reversal of scalar gradients in the middle of the PBL. Because for $A$ significantly larger than 0 a countergradient flux (or an increasing lapse rate) seems unphysical, one is hard pressed to justify values of $\gamma_{c} \kappa$ larger than six, which is about the magnitude of values in common use. This conclusion supports some previous work on the subject (e.g., Holtslag and Moeng 1991), as it further indicates that for the diffusivities modeled by (12) with $\kappa=$ $0.675, \gamma_{h} \approx 5$ is more appropriate than the larger values that are sometimes used.

\section{c. Integral scaling}

Cuijpers and Holtslag (1998) argue that the nonlocal fluxes are more appropriately scaled by integral fluxes. For the special case of conserved quantities this implies that in a quasi-steady state (where the fluxes are linear and the integral can be evaluated analytically), the scale value for a scalar flux $\overline{w c}$ is simply $\overline{w c}_{*}=\left(\overline{w c}_{0}+\overline{w c}_{z}\right) / 2$, which for $\overline{w c}_{z_{i}}=A \overline{w c}_{0}$ implies that $\overline{w c}_{*} / \overline{w c}_{0}=(1+A) / 2$.

This revised scaling, in that the entrainment fluxes now explicitly appear in the scaling flux, results in a slightly different class of solutions. Namely those that satisfy 


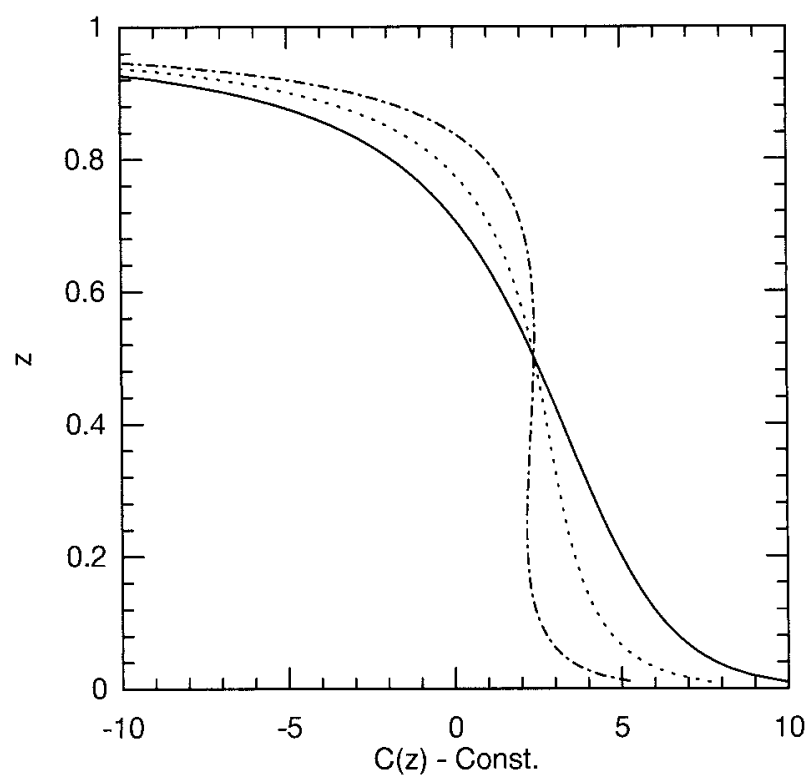

FIG. 6. Quasi-steady profiles for a $C$ scalar with $A=0.5$. Designed to mimic a moisture variable. Solid, dashed, and dashed-dotted lines, respectively, plot solutions for $\left(\gamma_{c}, \kappa\right)=(0,0.675),(5,0.675)$, and $(10,0.675)$.

$$
\frac{d C}{d z}=\frac{\gamma_{c} \kappa z(1-z)^{2}-\frac{2}{1+A}(1-z+A z)}{\kappa z(1-z)^{2}}
$$

as opposed to (14). Following the path of the previous analysis, let us consider zeros of this new equation, that is, zeros of a function

$$
\begin{aligned}
f_{2}\left(z ; A, \gamma_{c} \kappa\right)= & \gamma_{c} \kappa z^{3}-2 \gamma_{c} \kappa z^{2}+\left(\gamma_{c} \kappa+2 \frac{1-A}{1+A}\right) z \\
& -\frac{2}{1+A}=0 .
\end{aligned}
$$

The zeros of $f_{2}$ are plotted in Fig. 7; note that

$$
f_{2}\left(\frac{1}{2} ; A, \gamma_{c} \kappa\right)=\frac{1}{8} \gamma_{c} \kappa-1,
$$

which is why, irrespective of the value taken by $A$, at $z=1 / 2$ the zeros are found at $\gamma_{c} \kappa=8$.

In general, Fig. 7 yields similar results to the previous analysis, with one important, and surprising exception: the prediction that for a pure "top-down" scalar there

\footnotetext{
${ }^{4}$ A top-down scalar is one for which $A \rightarrow \infty$ implying $\overline{w c}_{z_{i}}>0$ and $\overline{w c}_{0}=0$, and is the complement to a bottom-up scalar defined, which has the property that $\overline{w c}_{0}>0$ and $\overline{w c}_{z i}=0$. This way of looking at the profiles of scalars was introduced by Wyngaard and Brost (1984). Note that our analysis implies that the scaling of the top-down and bottom-up scalars differs (both are scaled by the same value, i.e., the average flux) from the original proposals in situations when the generalized scalar is composed of both a top-down and bottom-up component.
}

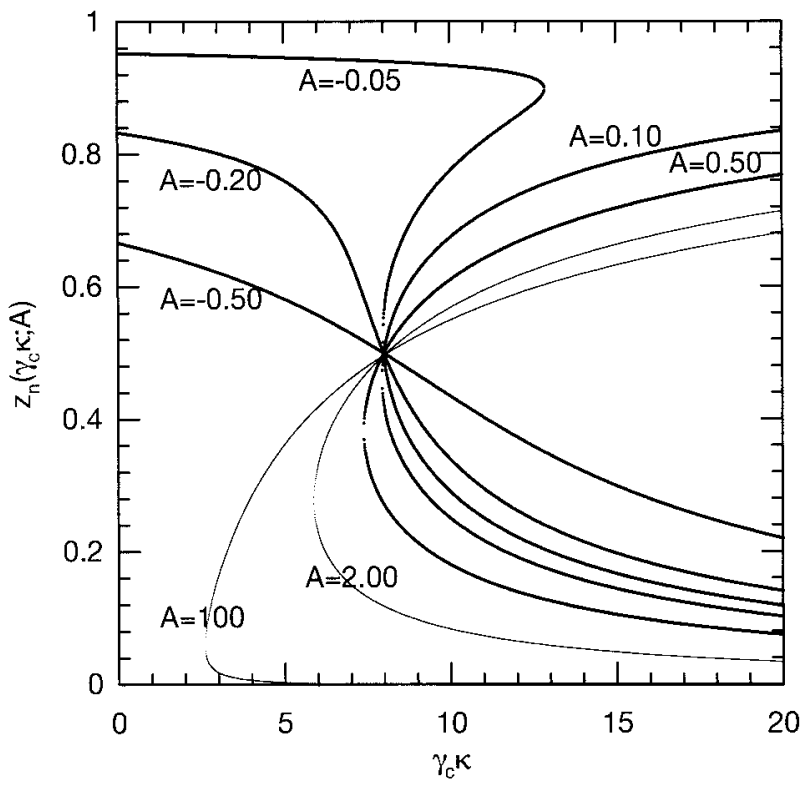

FIG. 7. Solutions to (19) for different values of $A$ as labeled. Note that in comparison to Fig. 5a we have added two solutions (denoted by thin lines) for $A=2$ and $A=100$.

exists some $z \in[0,1]$ for which $f_{2}(z)=0$. In physical terms, this says that the gradient of the profile vanishes somewhere inside the PBL. Because the flux is positive definite (everywhere greater than zero for $z \in[0,1]$ ), the $K$-profile model with integral scaling predicts that the concept of an eddy diffusivity does not hold for topdown scalars. This is not the case for the $K$-profile model scaled by surface convective fluxes; in that case the nonlocal term vanishes for top-down scalars. This result also differs from previous analyses of LES (e.g., see the discussions in Wyngaard 1987; Wyngaard and Weil 1991, and references therein) in which eddy diffusivities are found to be well defined for pure top-down scalars.

In a similar vein, we note that the similarity functions for bottom-up and top-down scalar gradients, denoted by $g_{b}(z)$ and $g_{t}(z)$, respectively, are just the limits of (18) as $A$ goes to 0 and $\infty$, respectively, that is,

$$
\begin{aligned}
& g_{b}(z)=\gamma_{c}-\frac{2}{\kappa z(1-z)} \\
& g_{t}(z)=\gamma_{c}-\frac{2}{\kappa(1-z)^{2}},
\end{aligned}
$$

from which it is apparent that $g_{t}(1-z) \neq g_{b}(z)$, that is, the scaling implies transport asymmetry between topdown and bottom-up scalars, where the source of this asymmetry is in the asymmetry in the velocity variance statistics (as represented by the profile of $K$ ).

\section{d. Relationships to previous work}

Many models (e.g., Large et al. 1994; Hong and Pan 1996) based on the approach pioneered by Troen and Mahrt (1986) write the dimensional forms $K_{h}^{d}$ and $\gamma_{h}^{d}$ : 


$$
\begin{aligned}
K_{h}^{d}\left(z^{d}\right) & =c_{1} w_{h}^{d}\left(z^{d}\right) z^{d}\left(1-z^{d} / z_{*}\right)^{2} \\
\gamma_{h}^{d}\left(z^{d}\right) & =\Gamma_{h} \frac{Q_{*}}{w_{h}^{d}\left(z^{d}\right) z_{*}},
\end{aligned}
$$

where $c_{1}$ is a constant that Large et al. (1994) set to unity; others (e.g., Troen and Mahrt 1986; Hong and Pan 1996) set $c_{1}=k$, where $k \approx 0.4$ is Kármán's constant. Here $\Gamma_{h}$ turns out to be proportional to $\gamma_{h}$ (as we show below); it is assigned various names in the literature, that is, $C$ by Troen and Mahrt (1986), $b$ by Hong and Pan (1996), and $C_{s}$ Large et al. (1994). The above equations differ from our previous formulation [e.g., Eq. (6)] through the introduction of the function $w_{h}^{d}$; a height-dependent surface-layer scale velocity defined by

$$
w_{h}^{d}\left(z^{d}\right)= \begin{cases}c_{2} u_{*} \phi_{h}^{-1}\left(\frac{z^{d}}{L}\right) & \text { if } z^{d}<\varepsilon z_{*} \\ c_{2} u_{*} \phi_{h}^{-1}\left(\frac{\varepsilon z_{*}}{L}\right) & \text { otherwise, }\end{cases}
$$

where $u_{*}$ is the friction velocity, $L=-z_{*} u_{*}^{3} /\left(k w_{*}^{3}\right)$ is the Monin-Obukhov length, $c_{2}$ is a constant, which is usually given the value of unity (e.g., Troen and Mahrt 1986; Hong and Pan 1996) or set to $k$ (Large et al 1994), $\varepsilon \approx 0.1$ is the nondimensional height of the surface layer, and $\phi_{h}$ is the nondimensional $\Theta$-profile, which in convective situations is generally given a form

$$
\phi_{h}\left(\frac{z^{d}}{L}\right)=\left(a_{h}-c_{h} \frac{z^{d}}{L}\right)^{-1 / 3},
$$

where $a_{h}$ and $c_{h}$ are empirical constants. From (25) and (26) it follows that

$$
w_{h}^{d}\left(z^{d}>\varepsilon z_{*}\right) \rightarrow c_{2}\left(k c_{h} \varepsilon\right)^{1 / 3} w_{*}
$$

in the convective limit.

Thus, nondimensionalizing $K_{h}^{d}$ and $\gamma_{h}^{d}$ by the convective scales leads to

$$
\begin{aligned}
& K_{h}(z>\varepsilon)=\frac{\kappa}{c_{1} c_{2}\left(k c_{h} \varepsilon\right)^{1 / 3}} z(1-z)^{2} \\
& \gamma_{h}(z>\varepsilon)=\frac{\Gamma_{h}}{c_{2}\left(k c_{h} \varepsilon\right)^{1 / 3}} .
\end{aligned}
$$

The point of all this should now be evident, namely that the forms for nonlocal fluxes and $K$ profiles in the existing literature relate to the nondimensional values discussed here in ways that depend on $c_{h}, c_{1}$, and $c_{2}$. Because neither Troen and Mahrt (1986), nor Hong and Pan (1996) write down the forms they use for $\phi_{h}$ we are unable to discern the value of $c_{h}$ that they use, consequently their models are difficult to compare quantitatively to values of $\gamma_{h}$ and $\kappa$ discussed here. Large et al. (1994) use $c_{h}=98.96$, which leads to $\kappa \approx 0.633$ and $\gamma_{h} \approx 10$. This combination leads to a value of $\gamma_{h} \kappa$, which is probably a factor of two too large..$^{5}$

\section{e. Surface-layer matching}

Equations (23)-(25) imply that within the surface layer $\kappa$ and $\gamma_{h}$ will have a dependence on $z$. However, because of the form of (9), surface-layer matching does not impact our solutions for the quasi-steady profiles above the surface layer, that is, for $z \geq \varepsilon$. In the surface layer, however, the additional $z$ dependence of $K_{h}$ and $\gamma_{h}$ results in a slightly modified equation for the gradient of $\Theta$ :

$$
\begin{aligned}
\left.\frac{d \Theta}{d z}\right|_{z \leq \varepsilon} & =\frac{\gamma_{h} \kappa z(1-z)^{2}-[(1-z)+A z]}{\kappa\left(\frac{z}{\varepsilon}\right)^{1 / 3} z(1-z)^{2}} \\
& =\gamma_{h}(\varepsilon / z)^{1 / 3}-\frac{\varepsilon^{1 / 3}}{\kappa z^{4 / 3}(1-z)}-\frac{A \varepsilon^{1 / 3}}{\kappa z^{1 / 3}(1-z)^{2}} .
\end{aligned}
$$

One could again integrate (31); here we satisfy ourselves with pointing out that the consistent application of surface-layer matching has no effect on the values of $z_{n}$, and only affects the near-surface values of the quasi-steady profile. The main effect of the revised surface-layer scaling is in accord with the desired effect; namely, it acts to force the near-surface values of $d \Theta / d z$ to scale with $z^{-4 / 3}$ as opposed to with $z^{-1}$. Further support for the surface layer forms used by Large et al. (1994) is provided by an analysis of LES results (Holtslag and Moeng 1991), which illustrates the tendency of $\gamma_{h}$ to increase toward the boundaries of the turbulent flow. However, it should be emphasized that because the nonlocal term affects the profile of the mean field throughout the boundary layer, it is not appropriate to match the bulk of the PBL to the surface layer in the absence of this term, which is why the nonlocal term is often set to zero for $z<\varepsilon$ (e.g., Large et al. 1994).

\section{Discussion}

\section{a. Previous studies}

Until now we have predominantly concerned ourselves with the mathematical properties of quasi-steady solutions to (4). In the introduction we claimed that such an analysis could help form the basis for better interpretations of sensitivity experiments conducted with PBL models that make use of (2). Consider, for example, the sensitivity studies described by Hong and Pan (1996) where they examined the response of the National Cen-

\footnotetext{
${ }^{5}$ Note that $\gamma_{h} \kappa$ implies a value of $z_{n} \approx 0.2$, which is not evident in the profile of Fig. 1 in Large et al. (1994); however, this is because that profile was constructed from a version of the KPP model used slightly different constants than what eventually were advocated for in the paper (W. G. Large 1998, personal communication).
} 
ter for Environmental Prediction's Medium-Range Forecast Model to changes in the boundary layer formulation. In particular they looked at how changes to the $K$-profile model and the nonlocal flux formulation affected forecasts of the PBL structure during the First International Satellite Land Surface Climatology Project (ISLSCP) Field Experiment. They found that the nonlocal term leads to a better ventilated surface layer, and a more stable upper boundary layer (for the case of $\Theta$ ) and a better mixed overall profile for the case of moisture. This is in accord with what one would expect based on the analytic, quasi-steady solutions. Thus our results support the premise underlying the analysis of Hong and Pan (1996), namely, that the profiles derived from the operational model result directly from changes made to the PBL parameterization.

Hong and Pan (1996) also show that increasing the exponent in the formulation of $K$ has an effect similar to reducing the magnitude of the nonlocal term. To understand this let us define $K_{p}=\kappa z(1-z)^{p}$, and recall that Hong and Pan (1996) compared solutions with $p$ $=2$ and $p=3$. Because $K_{3}^{\uparrow} \approx 0.7 K_{2}^{\uparrow}$ [recall the definition of $K^{\uparrow}$ from (13)], and because the location of the neutral point of the quasi-steady profile depends on $\gamma K^{\uparrow}$, reducing the maximum value of $K$ has an effect similar to reducing the value of the nonlocal term by a similar amount. Thus our analysis indicates that changing from $K_{2}$ to $K_{3}$ (in their forecast model) affects the solution in three different ways: (i) the shape of the profile is changed, which appears to be the desired effect of changing $p$, and is in our view a minor one; (ii) the magnitude of the $K$-profile is changed; and (iii) the behavior of the nonlocal term is changed due to the change in $K$.

\section{b. Sensitivities in a climate model}

Holtslag and Boville (1993) examine the sensitivity of a climate model to changes in the PBL scheme; however, they only report on differences between a nonlocal PBL scheme and a local PBL scheme, where the local scheme determines the mixing coefficients based on local properties of the state variables and the nonlocal scheme diagnoses a boundary layer depth (which is usually several levels) and diagnoses mixing properties based on the bulk properties of the PBL. Although the nonlocal scheme makes use of a variety of internal assumptions (such as the role of nonlocal processes), an examination of these issues was not an objective of the Holtslag and Boville study.

But we can touch on such issues here. We do so by comparing two 1-yr integrations of the standard CCM version 3.2 with fixed SSTs (Kiehl et al. 1996). In the first calculation we used the standard PBL package (which sets $\gamma_{h}=\gamma_{c}=10$ ), while in the second calculation we eliminate nonlocal processes by setting $\gamma$ $=0$. It is well beyond the intended scope of this paper to go into detail with regard to the differences between these simulations. However, some scalar measures of the overall response of the calculations to the presence of the nonlocal term are conveniently-if not definitively-interpreted in light of our above results. Namely, by analyzing the diagnostic outputs of the CCM, we find that in the absence of the nonlocal fluxes the globally averaged PBL depth over the last 3 months of the calculation increases by about $10 \%$. Furthermore, surface sensible heat fluxes are reduced by about $5 \%$.

Both the PBL height response, and the surface flux response, are consistent with the fact that in helping to better mix (or even stabilize) the outer layer, the PBL model must (in order to conserve heat and moisture) better ventilate the surface layer. Because the procedure for diagnosing the PBL depth depends on the temperature at the lowest model level (cf. Holtslag and Boville 1993), a less ventilated surface (in the absence of nonlocal processes) is consistent with a corresponding reduction in surface fluxes and an increase in PBL depth. This effect of the nonlocal term on the PBL depth diagnoses has also been noted by Beljaars and Viterbo (1998), who further point out that such an effect could impact the entrainment rate predictions by the model. Overall, our results are consistent with results from earlier analyses, which looked at these questions less directly (e.g., Holtslag and Boville 1993; Holtslag et al. 1995), in that it suggests that dynamically, the important consequence of (2) is that in attempting to better mix (or even stabilize) the upper part of the PBL, the nonlocal flux is forced to better ventilate the surface layers (see, e.g., Figs. 5b or 6), which thus can directly affect the state of the lowest model level and hence both the surface fluxes and (at least so far as the state of the lowest level is tied to a diagnoses of the PBL depth) entrainment fluxes (Beljaars and Viterbo 1998).

\section{c. Convergence and efficiency}

Our above analysis is useful in at least two other ways. First, analytic solutions can be used to diagnose directly the diffusive tendencies in a numerical integration. Second, the simple formulation of our problem [as per (4)] allows us to explore numerically how a coarsening of the vertical resolution affects the solution.

In doing the latter, we compare integrations of (4) using different vertical grids for a box with $z_{*}=1000$ $\mathrm{m}$, a fixed surface heat flux, $Q_{*}=0.2 \mathrm{Km} \mathrm{s}^{-1}$, and $A$ $=-0.2$. Numerically, we solve the system of equations using a hybrid backward implicit solver, in that (4) is solved using central differences of $\Theta$ valid at time $t+$ $\Delta t$ and values of the diffusivities valid at time $t$. In comparing simulations with $6,24,96$, and 384 levels (Fig. 8) we note two aspects of the solutions worth commenting on. First, similar to what was found in a less idealized setting by Holtslag et al. (1995), we find that for the most part, the relatively coarse representation of the layer does a good job in representing the vertical structure of the solution. Second, as the dis- 


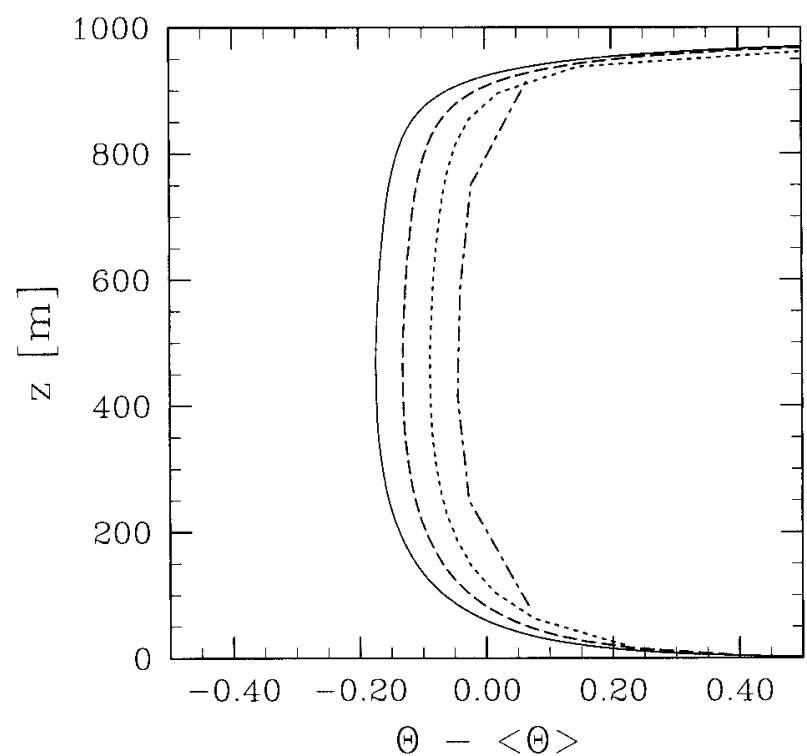

FIG. 8. The $\Theta(z)$ profiles for an initially well-mixed layer after $10800 \mathrm{~s}$ for four different discretizations. Here $N=384$ (solid), $N$ $=96$ (dashed), $N=24$ (dotted), $\mathrm{N}=6$ (dashed-dotted). All integrations are integrated as described in the text, and are checked to ensure they conserve $\Theta$ exactly, and thus have identical mean values $\langle\Theta\rangle$. Note that this follows from the fact that the linearly extrapolated profiles all cross in the surface layer.

cretization is refined (i.e., as we add more levels), the mid-PBL temperature becomes smaller and smaller, with no sense of convergence. Because the numerics conserve $\Theta$ to machine accuracy (we use 8-byte words), the reduction in the mid-PBL values of $\Theta$ with increasing refinement, is compensated for by higher values near the boundaries.

Mathematically, further insight into the nature of the discrete solutions can be gained by considering the behavior of

$$
\bar{\Theta}=\frac{1}{b-a} \int_{a}^{b} \Theta(z) d z,
$$

that is, the average value of $\Theta(z)$ in the interval $(a, b)$. The $N$-level discretization of this equation is simply

$$
\bar{\Theta}=\frac{1}{N} \sum_{i=1}^{N} \Theta\left(z_{i}\right)
$$

where

$$
z_{i}=a+\frac{b-a}{N} i-\frac{b-a}{2 N} .
$$

If we decompose this series into three separate series,

$$
\bar{\Theta}=\bar{\Theta}^{\gamma}+\bar{\Theta}^{A}+\bar{\Theta}^{Q_{0}},
$$

where the three terms correspond to the three processes in (16), that is, nonlocal fluxes $\left(\bar{\Theta}^{\gamma}\right)$, entrainment fluxes $\left(\bar{\Theta}^{A}\right)$, and surface fluxes $\left(\bar{\Theta}^{Q_{0}}\right)$, we can analyze the convergence of each process as $N$ increases. In so doing we find that $\bar{\Theta}^{\gamma}$ is independent of $N$ and that $\bar{\Theta}^{Q_{0}}$ con- verges rapidly with $N$. By rapidly we note that the discrete approximation of $\bar{\Theta}^{Q_{0}}$ in the surface layer (i.e., $a$ $=0$ and $b=0.1$ ) is, for $N=1$, within $10 \%$ of its convergent value, increasing to nearly $2 \%$ accuracy as $N$ increases to 5 .

The real difficulty lies with our formulation of the entrainment term. Inspection of (16) shows that $\bar{\Theta}^{A}$ diverges logarithmically due to the fact that $K_{h}$ vanishes as $(1-z)^{2}$ as $z \rightarrow 1$. As a result, discrete approximations to $\bar{\Theta}^{A}$ vary as $\ln (N)$. Because energy must be conserved, the constant of integration must compensate; that is, in the discrete case solutions are offset by an amount that depends on $N$ to compensate for the singularity in the $\Theta^{A}$ profile as $z \rightarrow 1$. This behavior is evident in Fig. 8, where the profiles are beginning to cross near the upper boundary, and where the offset is by equal amounts in the interior for successive quadruplings of the number of points. If instead we let $K$ go to zero less rapidly as $z \rightarrow 1$, or if we do not let $K$ go identically to zero at $z$ $=1$ [but instead match it to some interior eddy (or molecular) diffusivity (e.g., Large et al. 1994)] the undesirable behavior of the solutions is eliminated; furthermore, it can be shown that discrete representations of the analytic solutions (which the time-stepped equations want to approach) are capable of representing the analytic structure of the PBL with very few points.

\section{d. Physical issues}

\section{1) A PHYSICAL INTERPRETATION}

Although we have taken a predominantly mathematical point of view, this is not without its physical implications. For instance, our analysis shows that the $K$-profile models with nonlocal fluxes can be thought of as a relaxation model. That is, it tends to relax profiles of scalars toward a similarity form whose nondimensional shape is implicitly determined both by the shape of the $K$-profile, and the choice of scaling.

To see this more clearly, recall that we have shown how a similarity solution exists (which for this section, and for some scalar $C$, let us denote by $C_{s}$ ), with the property that for a time-invariant forcing

$$
\frac{\partial}{\partial z}\left[K_{h}\left(\frac{\partial C_{s}}{\partial z}-\gamma_{h}\right)\right]=\chi_{0},
$$

where $\chi_{0}$ is a constant determined by the nonzero component of the boundary conditions. With out any loss of generality we can consider $C(z, t)$ to be linearly composed of a similarity component $C_{s}(z)$, a time-varying vertically averaged component $\langle C\rangle(t)$ (where we use angle brackets to denote the vertical averaging), and a deviation term $C^{\prime}(z, t)$ [not to be confused with the turbulent fluctuating term $c(x, y, z, t)]$ :

$$
C(z, t)=\langle C\rangle(t)+C_{s}(z)+C^{\prime}(z, t) .
$$

Note that by definition, $\left\langle C^{\prime}\right\rangle=\left\langle C_{s}\right\rangle=0$. In the absence of large-scale advective fluxes, or sources or sinks of 
$C$, its governing equation is equivalent in form to (4), which upon application of (36) results in

$$
\frac{\partial}{\partial t} C(z, t)=\frac{d}{d t}\langle C\rangle+\frac{\partial C^{\prime}}{\partial t}=\chi_{0}+\frac{\partial}{\partial z}\left(K_{h} \frac{\partial C^{\prime}}{\partial z}\right) .
$$

But by definition $\chi_{0}=d\langle C\rangle / d t$, so that (37) simplifies to the following equation for the deviations from the similarity profile:

$$
\frac{\partial C^{\prime}}{\partial t}=\frac{\partial}{\partial z}\left(K_{h} \frac{\partial C^{\prime}}{\partial z}\right)
$$

Multiplying (38) by $C^{\prime}$ and taking the vertical average, allows us to form an evolution equation for the integrated variance of the deviation from the similarity profile, that is, the mean-square deviation, as a function of time,

$$
\frac{d}{d t}\left\langle\left(C^{\prime}\right)^{2}\right\rangle=\left.K_{h} \frac{\partial\left(C^{\prime}\right)^{2}}{\partial z}\right|_{0} ^{1}-\left\langle 2 K\left(\frac{\partial C^{\prime}}{\partial z}\right)^{2}\right\rangle .
$$

Because $K$ is positive semidefinite, the second term on the rhs unambiguously acts to dissipate $\left(C^{\prime}\right)^{2}$. The first term on the rhs is the integrated transport term; it reflects the fact that the only source of deviations is at the boundaries, but because the boundary conditions for the deviation component of the profile are zero by definition, this term must vanish.

Equation (39) expresses the fact that what is shown in Fig. 3 holds in general. Namely, that given an initial profile, and fixed boundary conditions, the PBL model considered here, unambiguously damps deviations from an implied similarity profile. As a result, under certain conditions, the model can be thought of as a way of relaxing the PBL profile of scalars to an implicitly defined similarity profile on a convective timescale. Thus in a sense, this class of models can be seen as similar, albeit more general, than that proposed by Betts (1995). This interpretation is probably true of a broader class of PBL models (excluding of course mixed-layer models, where the relaxation is assumed to be infinitely fast); what we have done here is merely demonstrate this point, along with the fact that in our case the form of this similarity profile is determined (but readily discernible) by the assumptions inherent in the formulation of the model, that is, the chosen shape of the $K$ profile, and the choice of scaling. Given this interpretation, one could view the nonlocal term less in terms of physical processes, and more as a simple method for introducing a larger class of similarity profiles toward which the boundary layer will be relaxed.

\section{2) Physical foundations And the IMPortance OF TESTS}

Heretofore we have largely accepted the $K$-profile framework as given. As indicated in the introduction, this reflects its wide use in the larger-scale modeling community, rather than its carefully demonstrated generality, or the lack of question among micrometeorologists or turbulence theorists regarding its merits. Indeed, this basic framework for modeling the fluxes within the PBL is mostly corroborated only on the basis of a relatively small number of relatively low-resolution large-eddy simulations in limited regimes of parameter space. ${ }^{6}$ Other theories, or models, for the fluxes in the PBL exist and have varying degrees of support.

For instance, in the introduction we noted that the original motivation for introducing a nonlocal flux was that of Ertel, based on the physical premise of biased starting plumes in convective flows. Subsequently Wyngaard (1987) proposed that changes in the mean field during parcel transit times could lead to transport asymmetry between top-down and bottom-up scalars in fields with skewed vertical velocity statistics. Wyngaard's idea was examined further, and formalized into an analytic model of a flux in which a nonlocal component is proposed to give an equation similar to (1) but with $N$ proportional to the flux gradient and the skewness of the vertical velocity (Wyngaard and Weil 1991). Cuijpers and Holtslag (1998) examined the ability of the Wyngaard and Weil model to describe results from LES, and found that in some situations a simple application of the proposed model failed to capture the behavior of the scalar statistics-largely because in the three flows simulated by Cuijpers and Holtslag the transport asymmetries in the scalar fluxes were poorly correlated with the skewness in the distribution of vertical velocities.

In contrast to the models discussed in the preceding paragraph, the nonlocal model that we have analyzed in this paper is largely developed without regard to a specific physical process, but instead based on what one might term pseudo- or LES empiricism. That is by analyzing large-eddy simulations Holtslag and coworkers have noted that the dimensional budget equation for the flux of some scalar $c$, namely,

$$
\frac{\partial \overline{w c}}{\partial t}=-\overline{w w} \frac{\partial C}{\partial z}+\frac{g}{\theta_{0}} \overline{\theta c}-\frac{\partial \overline{w w c}}{\partial z}-\frac{1}{\rho_{0}} c \overline{\frac{\partial p}{\partial z}},
$$

can, at least for some convectively driven situations in a quasi-steady state, be written in a highly parametric form as

$$
\frac{\partial \overline{w c}}{\partial t}=-\overline{w w} \frac{\partial C}{\partial z}+\eta\left(\frac{z}{z_{i}}\right) \frac{w_{*}^{2} C_{*}}{z_{*}}-\frac{\overline{w c}}{\tau} \approx 0,
$$

where $\eta$ is a height-dependent shape function, and the buoyancy production and turbulent transport of flux and

\footnotetext{
${ }^{6} \mathrm{PBL}$ models based on the $K$-profile framework have been compared to field data (e.g., Vogelezang and Holtslag 1996; Holtslag et al. 1995), but these comparisons normally test the totality of the model behavior in poorly constrained environments, as a result differences between the data and the calculations are difficult to attribute to specific components of the model.
} 
a component of the pressure covariance term [i.e., the second, third, and part of the fourth term on the rhs of (40)] are collectively represented by the second term on the rhs above, while the remainder of the pressure covariance term is modeled as the ratio of the flux to a return to isotropy timescale $\tau$. In convectively driven flows Cuijpers and Holtslag (1998) found that by appropriately defining $C_{*}$ and letting $\eta$ be constant, the model of the flux implied by (41), that is,

$$
\overline{w c}=-(\overline{w w}) \tau \frac{\partial C}{\partial z}+\eta \tau \frac{w_{*}^{2} C_{*}}{z_{*}},
$$

which is similar ${ }^{7}$ to the one given by (2), could satisfactorily reproduce the results from LES. However, we should not forget, that LES has been used here as a tool to make simple pseudoempirical statements about the character of second-order budgets in a limited range of circumstances. And while such an approach is by design able to reproduce many aspects of the flows from which it was derived, it is not at all certain that it will lead to results that are accurate in general.

For this reason it is especially important to continue to test or corroborate (using either field or laboratory data) the predictions of this model. To the extent that (2), (12), and $\gamma_{c}=$ constant are a faithful reproduction of the behavior of LES, attempts at corroboration will also, in a sense, be a test of LES. And while we have not endeavored to physically analyze or test the physical basis of the model here, it is our belief that an analysis such as ours, which illustrates in rich detail the basic properties of a class of nonlocal $K$-profile models, provides an indispensable context (or foundation) for future tests.

\section{Summary and conclusions}

For common forms of the eddy diffusivity and the nonlocal heat flux, we show how the nondimensional magnitude of these two terms affects the quasi-steady structure of scalars in the PBL. This is done by analytically solving for the profiles of conserved scalars in a quasi-steady state. The assumption of a quasi-steady state is a key one in facilitating our derivations, but it is not overly limiting in that most analyses used to justify models of the form we consider are themselves based on the quasi-steady assumption. The analytic solutions are useful in a number of respects:

1) They demonstrate how different processes contribute to the quasi-steady nondimensional profiles of heat and/or other scalars.

2) They demonstrate that there is only a limited range of values for $\gamma_{h} \kappa$ that lead to physical profiles across a reasonable range of parameter space. Too large a

\footnotetext{
${ }^{7}$ The equivalence is shown if we define $K(z)=(\overline{w w}) \tau$ and $K(z) \gamma$ $=\eta \tau w_{*}^{2} C_{*} / z_{*}$.
}

value of $\gamma_{h} \kappa$ can lead to superadiabatic $\Theta$ profiles near $z_{*}$, fluxes that already begin to become countergradient below $0.3 z_{*}$, and moisture profiles that increase with height. If $\gamma_{h} \kappa$ is too small, then the nonlocal flux will not be allowed to sufficiently contribute to the mixing within the PBL.

3) They demonstrate how the similarity structure of topdown an bottom-up scalars depends significantly on the choice of flux scale. Scaling fluxes by their vertically averaged value, as proposed by Cuijpers and Holtslag (1998), forces the nondimensional profiles of top-down scalars to have a neutral point somewhere in the PBL, a result that conflicts with previous research, and the results of the same model where the flux is instead scaled by its surface valuethereby drawing our attention to an apparent lack of consensus on these fundamental and important issues.

4) They demonstrate that most $K$-profile models can be thought of as relaxation models, wherein the equilibrium profile toward which the boundary layer is relaxed is determined by the parameters of the models and the choice of convective scales.

Although most of our analysis assumed simple forms for both the eddy diffusivity and the nonlocal flux, we showed how it could be extended to examine the effect of including surface-layer matching in the derivations for $K$ and $\gamma$. In so doing we found that such matching has no effect on the bulk of the PBL (i.e., above the surface layer), and thus need not affect the location of the neutral points of the $\Theta(z)$ profile.

The analytic results facilitate interpretations of results from more complicated models. Our cursory analysis of a climate simulation suggests that the dynamically important aspect of the nonlocal flux is its ability to help ventilate the surface layer, thereby enhancing surface fluxes. In models that diagnose the depth of the mixedlayer based on the temperature at the first model level above the ground, enhanced surface layer ventilation may lead to the diagnosis of shallower boundary layers. As noted previously (e.g., Holtslag et al. 1995), our results support the conclusion that in many implementations an important (and unintended) effect of the nonlocal flux is its ability to modulate the diagnosis of the boundary layer height, and thus the determination of the entrainment flux at the top of the PBL.

Last, numerical integrations suggest that given an accurate estimate of the depth of the PBL, as well as the fluxes into it from above and below, relatively few vertical modes are needed to well represent its vertical structure. However, letting $K$ go to zero as $(1-z)^{n}$, where $n>1$, results in a singularity at $\Theta(z=1)$. This singularity tends to complicate the convergence tests, and suggests that in principle $K$ should not be allowed to vanish at $z=1$ but instead should be matched to an interior eddy, or molecular, diffusivity. 
Acknowledgments. Much of this work was carried out while the author was a postdoctoral fellow with the Advanced Study Program at NCAR, which is acknowledged for providing a wonderful intellectual environment in which to work. Revisions were made while the author was visiting the Max-Planck-Institut für Meteorologie in Hamburg, Germany, as a fellow of the Alexander Humboldt Foundation. It is also a pleasure to thank Chin-Hoh Moeng, and in particular to acknowledge her stubbornness, which to a large degree motivated this investigation. Bert Holtslag, Bill Large, and John Wyngaard also made a great number of very constructive suggestions that greatly improved this manuscript as well as my understanding of the basic issues at hand. Larry Mahrt, Arthur Petersen, Jimmy Dudhia, and two anonymous reviewers also made helpful suggestions that led to improvements in the manuscript. Last, the Tex project, the Free Software Foundation (GNU), and James Theiler (xyplot) are thanked for providing the word processor, text editor, and plotting routines used in this analysis.

\section{REFERENCES}

Beljaars, A., and A. K. Betts, 1992: Validations of the boundary layer scheme in the ECMWF model. Proc. ECMWF Seminar on Validation of Models Over Europe, Vol. 2, Shinfield Park, Reading, United Kingdom, ECMWF.

—, and P. Viterbo, 1998: Role of the boundary layer in a numerical weather prediction model. Clear and Cloudy Boundary Layers, A. A. M. Holtslag and P. G. Duynkerke, Eds., Elsevier, 287304.

Betts, A. K., 1995: A lagged mixing parameterization for the dry convective boundary layer. Mon. Wea. Rev., 123, 1912-1915.

Cuijpers, J. W. M., and A. A. M. Holtslag, 1998: Impact of skewness and nonlocal effects on scalar and buoyancy fluxes in convective boundary layers. J. Atmos. Sci., 55, 151-162.

Deardorff, J. W., 1966: The counter-gradient heat flux in the lower atmosphere and in the laboratory. J. Atmos. Sci., 23, 503-506.

- 1972: Theoretical expression for the countergradient vertical heat flux. J. Geophys. Res., 77, 5900-5904.
Ertel, H., 1942: Der vertikale Turbulenz-Wärmestrom in der Atmosphäre. Meteor. Z., 59, 250-253.

Holtslag, A. A. M., and C.-H. Moeng, 1991: Eddy diffusivity and countergradient transport in the convective atmospheric boundary layer. J. Atmos. Sci., 48, 1690-1698.

_ , and B. A. Boville, 1993: Local versus nonlocal boundary-layer diffusion in a global climate model. J. Climate, 6, 1825-1842.

—, E. van Meijgaard, and W. C. de Rooy, 1995: A comparison of boundary layer diffusion schemes in unstable conditions over land. Bound.-Layer Meteor., 76, 69-95.

Hong, S.-Y., and H.-L. Pan, 1996: Nonlocal boundary layer vertical diffusion in a medium-range forecast model. Mon. Wea. Rev., 124, 2322-2339.

Kiehl, J. T., J. J. Hack, G. B. Bonan, B. A. Boville, B. P. Briegleb, D. L. Williamson, and P. J. Rasch, 1996: Description of the NCAR Community Climate Model (CCM3). NCAR Tech. Rep. NCAR/TN-420+STR, 152 pp. [Available from National Center for Atmospheric Research, P.O. Box 3000, Boulder, CO 803073000.]

Large, W. G., J. C. McWilliams, and S. C. Doney, 1994: Oceanic vertical mixing: A review and a model with a nonlocal boundary layer parameterization. Rev. Geophys., 32, 363-403.

Mailhôt, J., and R. Benoit, 1982: A finite-element model of the atmospheric boundary layer suitable for use with numerical weather prediction models. J. Atmos. Sci., 39, 2249-2266.

Priestley, C. H. B., 1959: Turbulent Transfer in the Lower Atmosphere. University of Chicago Press, $130 \mathrm{pp}$.

—, and W. C. Swinbank, 1947: Vertical transport of heat by turbulence in the atmosphere. Proc. Roy. Soc. London, Ser. A, 189, $543-561$.

Sutton, O. G., 1953: Micrometeorology. McGraw-Hill, 333 pp.

Troen, I. B., and L. Mahrt, 1986: A simple model of the atmospheric boundary layer: Sensitivity to surface evaporation. Bound.-Layer Meteor., 37, 129-148.

Vogelezang, D. H. P., and A. A. M. Holtslag, 1996: Evaluation and model impacts of alternate boundary-layer height formulations. Bound.-Layer Meteor., 81, 245-269.

Wyngaard, J. C., 1987: A physical mechanism for the asymmetry in top-down and bottom-up diffusion. J. Atmos. Sci., 44, 10831087.

_ , and R. A. Brost, 1984: Top-down and bottom-up diffusion of a scalar in the convective boundary layer. J. Atmos. Sci., 41, $102-112$.

— , and J. C. Weil, 1991: Transport asymmetry in skewed turbulence. Phys. Fluids, A3, 155-162. 